University of Nebraska - Lincoln

DigitalCommons@University of Nebraska - Lincoln

1985

\title{
Two Modal Action Patterns with a Continuous Temporal Distribution
}

\author{
Alan B. Bond \\ University of Nebraska - Lincoln, abond1@unl.edu \\ George W. Barlow \\ University of California, Berkeley \\ William Rogers \\ University of California, Berkeley
}

Follow this and additional works at: https://digitalcommons.unl.edu/bioscibehavior

Part of the Behavior and Ethology Commons

Bond, Alan B.; Barlow, George W.; and Rogers, William, "Two Modal Action Patterns with a Continuous Temporal Distribution" (1985). Papers in Behavior and Biological Sciences. 52.

https://digitalcommons.unl.edu/bioscibehavior/52

This Article is brought to you for free and open access by the Papers in the Biological Sciences at DigitalCommons@University of Nebraska - Lincoln. It has been accepted for inclusion in Papers in Behavior and Biological Sciences by an authorized administrator of DigitalCommons@University of Nebraska - Lincoln. 
Z. Tierpsychol., 68, 326-334 (1985)

(C) 1985 Verlag Paul Parey, Berlin und Hamburg

ISSN 0044-3573 / InterCode: ZETIAG

Chesapeake Bay Center for Environmental Studies,

Smithsonian Institution, Edgewater, and Department of Zoology and Museum of Vertebrate Zoology,

University of California, Berkeley

\title{
Two Modal Action Patterns with a Continuous Temporal Distribution
}

\author{
By Alan B. Bond, George W. Barlow and William Rogers
}

With 3 figures

Received: April 9, 1984

Accepted: July 5, 1984

\begin{abstract}
Summary
Most methods of quantitative analysis of animal behavior assume that action patterns can be unambiguously classified into discrete, exclusive categories. This is not invariably the case. The digging behavior of the Midas cichlid (Cichlasoma citrinellum), for example, exhibits two functionally distinct modalities, scoop and pick, that intergrade continuously in form but are separable probabilistically. We present a technique for analyzing such behaviors that provides a reliable basis for formulating and verifying categories and allows a quantitative assessment of functional dissimilarity.
\end{abstract}

\section{Introduction}

An animal's stream of behavior is usually recorded and analyzed as if it consisted of a series of discrete, distinctive events. This is, in part, a consequence of the constraints of our analytical techniques, but it also reflects the tendency of human observers to segregate natural phenomena into discontinuous categories. It has long been recognized, however, that many types of behavior are resistant to such categorization. The original distinction between "appetitive" and "consummatory" actions was an attempt to separate the more variable and unpredictable classes of behavior from a set of precisely defined and invariant fixed action patterns, which were much easier to recognize and categorize. Even most fixed action patterns appear to be more variable than was originally imagined, however, leading BARLOw $(1968,1977)$ to propose that they be called "modal action patterns" (MAPs). 
This underlying temporal and spatial variability insures that any classification scheme will occasionally fail. The most difficult situation occurs when two functionally distinct MAPs grade almost imperceptibly into one another in form or duration. They may, in fact, be distinguishable only probabilistically, thereby frustrating all efforts to define and separate the behaviors in an objective fashion. Given the variability observed in natural behavior, the phenomenon of intergrading MAPs cannot be uncommon; it poses a significant practical problem for quantitative analysis, one that has not been satisfactorily addressed in the literature.

The digging behavior of cichlid fishes exhibits characteristic patterns that differ from one another mainly in the length of time that the gravel substrate is held in the mouth. In the brief, nearly instantaneous, acts that we have termed "picks" ("sifting" in HeILIGenberg 1965), the fish tilts forward, bites lightly at the bottom, and quickly returns to the horizontal position. On occasion, chewing or sifting movements ensue, followed by the ejection of debris. At the other temporal extreme, the fish tilts forward and plunges its mouth deeply into the substrate. It then returns to the horizontal, swims forward some little distance without chewing or sifting, and discharges the mouthful of gravel. We have called these longer-duration digging acts "scoops" ("carrying" in HeILIGENBERg 1965).

There appear to be two principal alternative functions involved in this behavior. Either the displacement of the gravel is in itself the primary goal of the action, as in the construction of nesting pits, or it is merely a secondary consequence of rooting about for food that may be buried in the gravel or adhering to it. Identification of the short-duration picks with foraging movements and the long-duration scoops with excavation behavior is, thus, intuitively appealing.

Testing the reality of this functional distinction, however, requires a means of discriminating the two action patterns on formal grounds. The use of contextual information in classifying the events would render meaningless any subsequent, objective test of their functional dissimilarity. The difficulty in this case is that there is nothing in the form of the behaviors themselves that assures an unambiguous classification. Although the extreme cases can readily be discriminated, the form of the behaviors intergrades smoothly and imperceptibly, and the categorization of events of an intermediate duration and appearance is by no means a simple task. We became concerned with the question of whether objective evidence could be adduced to support a functional distinction in these circumstances, whether there was a quantitative means of testing the intuitive functional correlations.

A recent study of the aggressive responses of these fishes to dummies of conspecific intruders (BARLOw et al. 1984) provided an extensive sample of the time course of digging behaviors. A procedure for analyzing the frequency distribution of event durations was developed and applied to this database.

Our primary assumption in this analysis was that the temporal continuum of digging acts was actually a compound distribution, consisting of two functionally distinct behaviors with distinguishable, though overlapping, prob- 
ability distributions. Our first requirement, therefore, was a procedure for partitioning the empirical distribution of digging events into two overlapping sets. The problem was approached heuristically, using an auxiliary source of duration data, rather than by fitting theoretical functions to the initial distribution.

The second requirement was an objective means for deriving a criterion for categorizing the digging events. Since the underlying distributions overlapped, no criterion could wholly avoid misclassification. Classification errors would contribute to the error variance and obscure the analysis of function and context, however. Consequently, we wished to obtain a criterion that minimized the frequency of errors for the given pair of distributions. Signal-detection theory (McNicol 1972) provides a simple means for determining criteria with just this property.

To avoid confusion, we will refer to the true, functionally distinct behaviors that are associated with the two underlying probability distributions in lower case letters ("picks" and "scoops"). Events that are categorized according to a duration criterion will be given with initial capitals ("Picks" and "Scoops").

Given the classification criterion, objective evidence that the two duration categories did refer to functionally distinct behaviors could then be sought. The technique of choice here was factor analysis, a means of statistical redescription of the correlation matrix of frequencies of events in terms of a smaller number of compound events or factors that are temporally, and therefore presumably contextually, homogeneous. Differences between event categories in their contribution to the factor structure could then be considered reasonable grounds for inferring a functional distinction.

\section{Materials and Methods}

The subjects were 12 adult Cichlasoma citrinellum, bred from stock originally captured in Lake Masaya, Nicaragua. 6 were of the normal cryptic coloration and 6 were goldcolored amelanic morphs (described in BarLow 1976). Half of the subjects of each color were males, and half were females. The subject fish ranged in size from 220 to $250 \mathrm{~g}$ and from 170 to $190 \mathrm{~mm}$ standard length. The experimental aquarium $(90 \times 60 \times 46 \mathrm{~cm}$ deep) contained one subject and 10 blinded juveniles (ca. $10 \mathrm{~g}$ each), half of which were goldcolored and half normal. The fish were fed twice daily with commercially available dried flakes or our own gelatin-based preparation (BARLOw and CAPPETO 1974). A more detailed account of the apparatus and treatment conditions is provided in BARLow et al. (1984).

In the course of a 2-h session each subject fish was exposed to 7 treatment conditions: five involved 1-min presentations of dummy fish of various sizes, and two employed blank controls. Following each treatment the sequence and duration of all MAPs was recorded continuously for $15 \mathrm{~min}$; to quantify short-term fluctuations in frequency of event, this interval was subsequently divided into three 5 -min observation periods. Each subject was' given one experimental session per day for 6 days in succession, varying systematically the order of treatment presentation. A total of 126 observation periods was thus accumulated for each of the 12 subjects, generating a pooled sample of 1512 periods or $126 \mathrm{~h}$ of observation.

An observer, seated behind a blind, entered the behavioral categories and contexts manually on a keyboard. Each keystroke generated distinctive signals, one when the key 
was depressed and another when it was released. Event durations for 9 behavioral categories (Bite, Butt, Charge, Flare, Tailbeat, Track, Quiver, Yawn, and Dig) were captured by holding down the appropriate key for as long as the behavior persisted. Durations were recorded on digital tape via a microprocessor with a real-time clock. The microprocessor could record up to 200 events/s; it thus registered with great accuracy slight variations in the duration of even the briefest depression of a key.

The primary focus of the study was aggressive behavior, comparing the frequency with which the four aggressive acts (Bite, Butt, Charge and Flare) were addressed to the different sizes of dummy fish and the different colors of blinded juveniles. It was therefore necessary to record, in addition to the event type and duration, the object toward which a particular aggressive action was directed. This information was supplied by "modifier" events encoded on a second set of keys. To record a charge by the subject fish toward one of the gold juveniles, for example, the observer. would first strike the "charge" key, holding it down for as long as the action persisted; when the action was completed, he would strike the "Gold" modifier key to indicate the context.

Modifier events only provided additional descriptors of the action. They therefore had no meaningful duration component: the appropriate key was merely strudk and released. As a consequence of the design and sensitivity of the recording system, however, a fortuitous record of the durations of these keystrokes was also obtained.

\section{Results}

\section{Characterization of the Frequency Distributions}

When the distribution of durations of digging events was plotted (Fig. 1), it became apparent that devising a means of separately characterizing the two component distributions would not be simple or straightforward. The underlying bimodality of the empirical distribution was almost completely obscured by the enormous disproportion in the relative frequencies of the two behaviors. The short-duration pick events were perhaps ten times as frequent as the longer-duration scoops, resulting in an apparently monophasic distribution with a rather irregular, extended tail. Separation of the component distributions therefore required a procedure for subtracting out the superabundance of short-duration picks to expose the features of the distribution of the less common long-duration scoops. A rough and approximate technique for accom-

Fig. 1: Frequency histogram of the durations of digging events. The total sample size was 29,124 acts, derived from $10.5 \mathrm{~h}$ of observation on each of 12 subjects

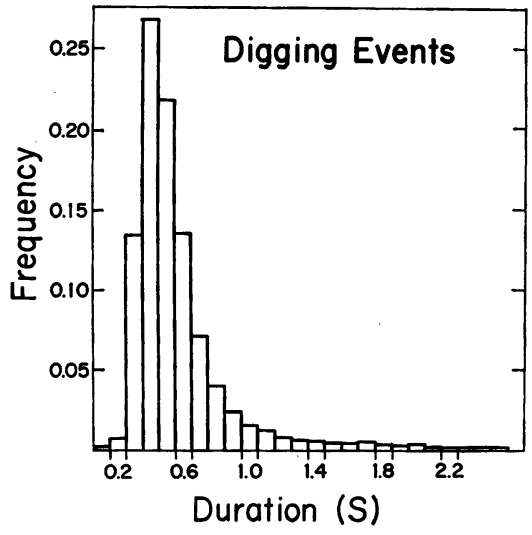




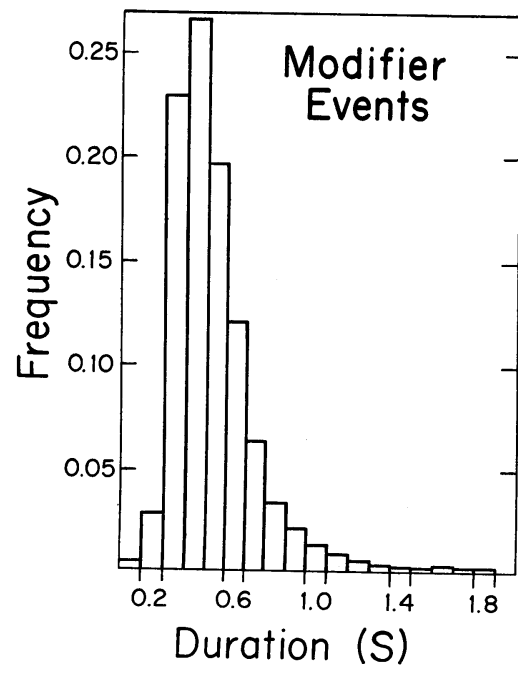

Fig. 2: Frequency histogram of the durations of modifier events, derived from a sample of 12,015 keystrokes

plishing this goal was made available by the fortuitous recording of the durations of modifier events.

Because the moment at which a modifier key was released was not dictated by the behavior of the fish, the duration of modifier events (Fig. 2) reflected only the unconstrained time course of the observer's key-pressing behavior. The distribution of digging events (Fig. 1) presumably included this observer behavior as one component of the variance, in addition to the components attributable to variation in the actual duration of picks and scoops.

The striking similarity in the shapes of the two curves (Figs. 1 and 2) led us to estimate that, because the duration of the majority of pick events was virtually instantaneous, their contribution to the variance of the aggregate distribution was likely to have been negligible, relative to that of the other two sources. By this reasoning, the shape of the pick distribution would have been essentially identical to that of the duration of modifier events, with the addition of a small positive displacement, representing the time required to verify that a pick event had terminated. Therefore the modifier distribution, appropriately displaced, could provide a rough approximation to the pick distribution and could be subtracted from the empirical dig histogram to reveal the distribution of the scoops.

The true displacement value was, of course, unknown, as was the proportion of the total sample of digging events that was assignable to the pick distribution. These two parameters were jointly optimized by minimizing the squared deviations of the fitted function from the observed values in the central peak of the dig curve $(0.2$ to $0.8 \mathrm{~s})$. The optimal displacement value was $40 \mathrm{~ms}$; the best-fit number of pick events was 27,357 , or $94 \%$ of the total. When the fitted distribution of pick events was subtracted from the empirical function, it left the difference histogram displayed in Fig. 3. This distribution is patently bimodal. 
Fig. 3: Difference histogram, indicating the estimated distribution function of scoops. Residues of the pick distribution that were edited off are shown with cross-hatchings. The sample size for the final, edited distribution was 1811 . The likelihood ratio associated with the first 18 duration bins is plotted with a dashed line; ratios for higher duration levels were off the scale of the graph. The decision criterion for separating Pick and Scoop categories is marked with an asterisk

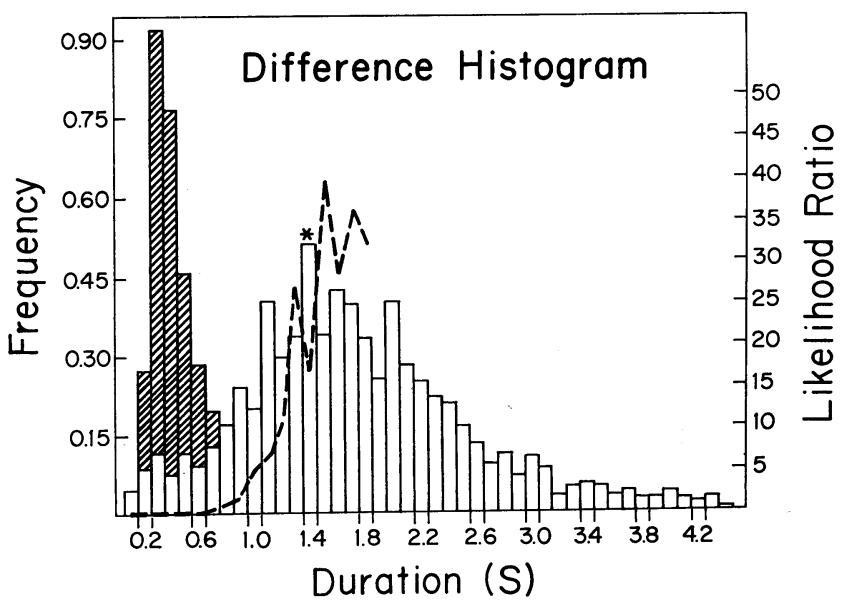

Separating the scoop distribution from the remaining pick events that filtered through the subtraction process was now a relatively minor task. We made a conservative assumption of symmetry, reflected the scoop distribution around its apparent median (1.6 s), and edited off the excess (shaded portion of the curve in Fig. 3). More sophisticated fitting techniques could have been employed, but they would have had only a negligible effect on the assessment of the criterion value.

\section{Derivation of the Criterion}

The decision rule that will maximize the number of correct classifications from two overlapping probability distributions is expressed in one of the fundamental theorems of signal-detection theory (McNicol 1972). If $P(x \mid p)$ is the conditional probability that the event duration will take the value $\mathrm{x}$, given that a pick has occurred, and $\mathrm{P}(\mathrm{x} \mid \mathrm{s})$ is the corresponding probability in the case of a scoop, then the odds ratio in favor of a classification of Scoop at the given duration is $\mathrm{P}(\mathrm{x} \mid \mathrm{s}) / \mathrm{P}(\mathrm{x} \mid \mathrm{p})$. In signal-detection theory, this is termed the "likelihood ratio", $1(x)$, and is used as the basis for developing a decision criterion, $\beta$. Any event with a duration associated with a value of $1(x) \leqslant \beta$ will be classified as a Pick; events with durations for which $l(x)>\beta$ will be termed Scoops.

It can be shown that the criterion that will discriminate two distributions most effectively is equal to the inverse ratio of the over-all probabilities of the events: $\beta=\mathrm{P}(\mathrm{p}) / \mathrm{P}(\mathrm{s})$. In this study, $\beta=27,357 / 1811=15.11$. Values of the likelihood ratio for the critical portion of the range of durations are graphed in Fig. 3. The first duration bin for which $1(x)$ exceeds $\beta$ is $1.3 \mathrm{~s}$. We have, therefore, chosen $1.4 \mathrm{~s}$ as the lower boundary of the Scoop category. This criterion falls at about the 39th percentile of the scoop distribution and the 99 th percentile of the pick distribution. The probability that an event will be properly categorized as a Scoop is, therefore, 0.77 , and $61.2 \%$ of the scoops will be correctly identified. 
Table 1: Factor matrix of frequency scores for all behaviors, following varimax rotation

\begin{tabular}{|l|c|c|c|c|c|}
\hline \multirow{2}{*}{ Behavior } & \multicolumn{5}{|c|}{ Factor } \\
\cline { 2 - 6 } & 1 & 2 & 3 & 4 & \multicolumn{1}{c|}{5} \\
\hline Bite & .742 & .175 & -.028 & .086 & -.112 \\
Butt & .157 & .568 & -.175 & -.105 & -.063 \\
Charge & .664 & .023 & .080 & .019 & .265 \\
Flare & .054 & -.009 & .011 & .059 & .552 \\
Tail Beat & .001 & .011 & .016 & .333 & .084 \\
Track & .043 & .018 & .013 & .085 & -.002 \\
Quiver & .039 & -.049 & -.048 & .250 & .000 \\
Yawn & .031 & -.033 & .596 & -.080 & .004 \\
Scoop & -.076 & -.010 & -.042 & .439 & -.004 \\
Pick & -.032 & -.544 & -.389 & -.121 & -.087 \\
\hline
\end{tabular}

* Coefficients $>0.25$ are italicized.

\section{Factor Analysis of Behavioral Frequencies}

Given this categorical division, the experimental data set provided frequency measures for ten different MAPs (including Pick and Scoop) for each of 1,512 observation periods. If our rough segregation procedure had yielded meaningful results, a factor analysis of the category frequencies should produce functionally or motivationally consistent groupings. We would, in addition, expect that substantial differences should be apparent between the patterns of factor loadings for Picks and Scoops, thereby providing empirical evidence of their functional dissimilarity. Using the SPSS Principal Factor procedure (PA2; NIE et al. 1975), we obtained five factors with eigenvalues greater than 1.0, accounting for a cumulative total of $62.4 \%$ of the variance. Varimax rotation to clarify the variable relationships yielded the matrix of factor loadings displayed in Table 1.

Factors 1, 2, and 5 appear to represent components of aggression, reflected in the large positive coefficients for attack and threat behaviors. The cluster of behaviors on Factor 4 - Tailbeat, Quiver, and Scoop - are all commonly observed during courtship, suggesting a sexual function for this grouping. Picks appear to be functionally distinct from the rest of the categories, loading predominantly on two bipolar factors (2 and 3). Pick and Scoop display no commonality in factor structure, which is fully consistent with the earlier, qualitative assessment of functional dissimilarities.

\section{Discussion}

The occurrence of intergrading action patterns in digging behavior emphasizes the ambiguity of the form of the behavior alone as an indicator of function. When the variability in the time course of an action is high, it may conceal the occurrence of functionally distinct temporal modes of behavior that are similar in form. This possibility should particularly be suspected when apparently similar behaviors are observed in different contexts. Under 
these circumstances, an objective analysis of the frequency distribution of durations of events may be required to substantiate the distinction.

The procedure outlined in this paper provides a reliable basis for formulating behavioral categories and assessing functional dissimilarity in continuously intergrading action patterns. Most features of the analysis appear to be generally applicable, although the optimal approach to estimating the distributions of the components must depend on the shape of the aggregate histogram and the availability of additional data. In cases of distinct bimodality, the two distributions could be fitted to appropriate mathematical functions (possibly log-normal or gamma distributions) or separated empirically either by eye or through the use of a smoothing algorithm. Alternatively, the two behaviors could be elicited experimentally in separate, controlled, contexts; the resulting frequency distributions could then be applied to the analysis of more complex situations.

The separation techniques used in this study do highlight a point often overlooked in ethological work. It is that the mechanical recording of a stream of behavioral events is not a purely objective account of the actions of an animal; it contains components of the behavior of the observer, as well. The influence of the observer can involve effects on the recorded time course of the behavior as well as the better known problems of data selection, misclassification, and reactivity. In many cases, it may be useful to incorporate explicit control features into the observational protocol that would allow reconstruction and separation of features of the behavior of the animal from the inadvertent consequences of the observer's interpretations and recording techniques.

\section{Zusammenfassung}

Die meisten Methoden quantitativer Verhaltensanalysen setzen eindeutige Klassifizierung der Verhaltensweisen in sich wechselseitig ausschließende Kategorien voraus. Die sind aber nicht immer gegeben. Das Graben des MidasCichliden Cichlasoma citrinellum wird in zwei funktional verschiedenen Weisen ausgeführt, „Schaufeln" und „Picken“, die der Form nach ineinander übergehen und nur statistisch unterscheidbar sind. Eine Analysetechnik für solche Fälle wird vorgestellt, die eine verläßliche Basis für eine Kategorienbildung bietet und eine quantitative Einschätzung der funktionalen Unterschiede dieser Verhaltensweisen erlaubt. Die nachfolgende Faktorenanalyse bestätigte die funktionale Unterscheidung der beiden Endhandlungen (Modal Action Patterns). Das Verfahren scheint allgemein anwendbar.

\section{Acknowledgements}

This research project was supported by National Sciences Foundation Grant BNS 7924081 to G. W. BARLOW. Funding for analysis of the data was provided, in part, by the Smithsonian Institution. We thank Axel MEYER for preparing the Zusammenfassung. 


\section{Literature Cited}

Barlow, G. W. (1968): Ethological units of behavior. In: The Central Nervous System and Fish Behavior. (Ingle, D., ed.) Univ. of Chicago Press, Chicago, pp. 217-237 • BARLow, G. W. (1976): The Midas cichlid in Nicaragua. In: Investigations of the Ichthyofauna of Nicaraguan Lakes. (Thorson, T. B., ed.) School of Life Sci., Univ. of Nebraska, Lincoln, pp. 333-358 - BARLow, G. W. (1977): Modal action patterns. In: How Animals Communicate. (SEвEOK, T. A., ed.) Indiana Univ. Press, Bloomington, Indiana, pp. 98-134 • Barlow, G. W., and R. V. Cappeto (1974): A food for all pisces. Buntb. Bull. 45, 19-21 • BARLow, G. W., W. Rogers and A. B. Bond (1984): Dummy-elicited aggressive behavior in the polychromatic Midas cichlid. Biol. Comport. 9, 115-130.

HEILIGENBERG, W. (1965): The effect of external stimuli on the attack readiness of a cichlid fish. Z. vergl. Physiol. 49, 459-464. London.

McNicol, D. (1972): A Primer of Signal Detection Theory. Allen and Unwin,

Nie, N. H., C. H. Hull, J. G. Jenkins, K. Steinbrenner and D. H. Bent (1975): SPSS: Statistical Package for the Social Sciences. McGraw-Hill, New York.

Authors' address: G. W. BARLow, Department of Zoology, University of California, Berkeley, California 94720, U.S.A. 